\section{Hautkrebsscreening: Schulung bringt Dermatologen nur wenig}

\author{
Dermatologen können ihre bereits guten Kenntnisse zu Hauttumoren durch \\ die Schulung zum Hautkrebsscreening kaum noch verbessern. Lediglich beim \\ Allgemeinwissen zum Screening haben sie in einer Studie noch zugelegt.
}

$\mathrm{N}$ ach der Krebs-FrüherkennungsRichtlinie des Gemeinsamen Bundesausschusses (G-BA) können bekanntlich außer Dermatologen auch Hausärzte und Internisten ihren Patienten eine Früherkennungsuntersuchung auf Hautkrebs anbieten und bei den gesetzlichen Krankenkassen abrechnen. Voraussetzung dafür ist eine von der zuständigen KV anerkannte achtstündige Fortbildung. Ob diese tatsächlich etwas bringt, haben Ärzte der Arbeitsgemeinschaft Dermatologische Prävention (ADP) aus Buxtehude untersucht.

Hierzu wurden Fragebögen von 573 Teilnehmern aus dem Jahr 2015 ausgewertet - $94 \%$ davon Allgemeinmediziner, die übrigen Dermatologen.

Den Ärzten wurden acht Abbildungen gezeigt, bei denen sie entscheiden mussten, ob es sich um kanzeröse Läsion handelt oder nicht. Vor der Schulung hatten die Hausärzte im Schnitt 3,9 Läsionen richtig eingeordnet, danach immerhin 5,3. Mit einem Cohen's-d-Wert von 1,0 ergab sich eine hohe Effektstärke für die Schulung. Anders sah es bei den Dermatologen aus - sie wussten auch schon vor der Schulung gut Bescheid und verbesserten sich nur noch von 5,2 auf 5,5 richtige Antworten - der Unterschied war nicht signifikant und mit einem Cohen's d von 0,2 auch nicht relevant.

Ähnliche Schulungseffekte ergaben sich bei der diagnostischen Genauigkeit: Hierzu mussten die Ärzte zwölf abgebildete Hautläsionen richtig benennen. Vor der Schulung gelang den Allgemeinärzten die richtige Diagnose im Schnitt bei 7,5 Abbildungen, danach bei 9,3. Die Dermatologen verbesserten sich dagegen nur wenig von 10,0 auf 10,2 richtige Diagnosen.

Allerdings war die Schulung auch für Dermatologen nicht gänzlich nutzlos, sie konnten immerhin ihr Allgemeinwissen zum Screening verbessern. Ein Komplex mit sieben Fragen zielte etwa auf die häufigste Hautkrebsform (Basalzellkarzinom) oder auf das nötige Equipment sowie die geeigneten Bedingungen für die Ganzkörperinspektion. Hier hatten die Fachärzte vor der Schulung im Mittel 5,4 von sieben Fragen richtig beantwortet, danach 6,3. Die Allgemeinärzte verbesserten sich von 5,5 auf 6,4 richtig beantwortete Fragen und lagen damit auf einem ähnlichen Niveau. In beiden Gruppen war der Unterschied signifikant und die Effektstärke hoch.

In einem weiteren Teil gaben die Ärzte an, wie sicher sie sich bei der Untersuchung und im Umgang mit den Patienten fühlen. Sowohl bei der Diagnose als auch bei der Patientenberatung legten die Allgemeinmediziner ausgehend von einem mittleren Niveau meist deutlich zu, nicht jedoch die Dermatologen - sie waren sich sowohl vor als auch nach der Schulung in allen Punkten sehr sicher.

Fazit: Unterm Strich profitieren von der Schulung also vor allem Hausärzte, Dermatologen eher weniger. Thomas Müller

Anders MP et al. Nationwide skin cancer screening in Germany: Evaluation of the training program. Int J Derm 2017; 56: 1046-51

\section{Milbenrhinitis: SLIT bei Jugendlichen effektiv}

\section{Was bringt die sublinguale spezifische Immuntherapie gegen Hausstaubmilben bei Jugendlichen mit einer entsprechenden allergischen Rhinitis? Die gepoolte Auswertung zweier großer Phase-III-Studien gibt Antworten.}

$M$ it der Entwicklung von Sublingualtabletten mit Hausstaubmilbenallergenen liegen erstmals auch für die Milbenrhinitis umfangreiche internationale Daten zu Wirksamkeit und Sicherheit vor. Insgesamt ergab sich ein positiver Effekt, der zur Zulassung der Milben-SLIT geführt hat. In einer retrospektiven Auswertung gepoolter Daten zweier großer in Nordamerika und Japan durchgeführten Studien sollten die Effekte nun noch einmal speziell in der Subgruppe von Jugendlichen analysiert werden.

Eingesetzt wurde eine schnellösliche Sublingualtablette mit einer 1:1-Mi- schung der Allergenextrakte von Dermatophagoides pteronyssinus und $D$. farinae in Dosierungen von einmal täglich $6 \mathrm{SQ}$ oder einmal täglich 12 SQ über jeweils ein Jahr. Von den 1.482 Teilnehmern der Studie A waren 189 Patienten im Alter zwischen zwölf und 18 Jahren, von den 946 Teilnehmern der Studie B 302. Der durchschnittliche totale kombinierte Rhinitisscore (TCRS) während der letzten acht Wochen der Behandlung bildete den primären Endpunkt in beiden Studien.

Die gepoolten Daten der Jugendlichen ergab für einmal täglich $12 \mathrm{SQ}$ im Vergleich zu Placebo eine TCRS-Verbesse- rung von $22 \%$. Auch der tägliche Rhinitissymptomscore, der tägliche Konjunktivitisscore und der tägliche Rhinitismedikationsscore besserten sich signifikant im Vergleich zu Placebo (alle $\mathrm{p}<$ 0,05). Häufigste Nebenwirkungen waren die bekannten leichten und reversiblen Nebenwirkungen im Mund-Rachenraum.

Fazit: Bei Jugendlichen mit einer allergischen Rhinitis gegen Hausstaubmilben bessert sich unter der einmal täglichen Applikation einer Sublingualtablette mit 12-SQ-HDM-Allergenextrakt der durchschnittliche totale kombinierte Rhinitisscore signifikant im Vergleich zu Placebo bei guter Verträglichkeit.

Dr. Barbara Kreutzkamp

Matsuoka T et al. Pooled efficacy and safety data for house dust mite sublingual immunotherapy tablets in adolescents. Pediatr Allergy Immunol 2017; 28: 661-7 